\title{
Lactancia materna y protección contra las infecciones respiratorias en los primeros meses de vida
}

\author{
MM. Bueno Campaña a , C. Calvo Rey ${ }^{\mathrm{b}}$, S. Jimeno Ruiz ${ }^{\mathrm{a}}$, \\ M. Faustino Sánchez ${ }^{b}, S$. Quevedo Teruel ${ }^{b}$, MÁ. Martínez Granero $^{a}$, \\ A. Delgado Iribarren`, I. Casas Flecha ${ }^{d}$ \\ ${ }^{a}$ Servicio de Pediatría. Hospital Universitario Fundación Alcorcón. Alcorcón, Madrid. España. \\ bservicio de Pediatría. Hospital Severo Ochoa. Leganés, Madrid. España. \\ 'Servicio de Microbiología. Hospital Universitario Fundación Alcorcón. Alcorcón, Madrid. España. \\ dLaboratorio de Gripe y Virus Respiratorios. Centro Nacional de Microbiología. \\ Instituto de Salud Carlos III. Madrid. España.
}

Fecha de publicación en Internet: 03 de mayo de 2011

\section{Resumen}

Introducción: entre las ventajas de la lactancia materna (LM) se incluye su papel protector ante infecciones respiratorias (IR).

Objetivos: describir el patrón de $L M$ de una cohorte de recién nacidos $(R N)$ y su protección contra las IR en el lactante.

Material y métodos: estudio prospectivo de 316 RN, de las áreas 8 y 9 de Madrid, desde el nacimiento mediante llamadas telefónicas quincenales durante el invierno, registrando datos clínicos y epidemiológicos. Si presentaban sintomatología compatible con IR se recogió aspirado nasofaríngeo.

Resultados: mil ochocientas sesenta y cinco llamadas (mediana: 4,2 [1-11]) y 106 visitas programadas. Al mes, el 56,1\% recibía $L M$ exclusiva; a los tres meses, el 39,4\%, y a los cinco meses, el 31,9\%. La LM exclusiva se mantuvo 65,1 días (desviación estándar: 43,49) y ningún factor se asoció a una mayor duración. Hubo 89 episodios de IR aguda. Los hermanos escolares multiplican por 1,74 (intervalo de confianza del 95\% [IC 95\% ]: 1,12-2,72) el riesgo de $I R$ en todos los estratos de duración de $L M$ exclusiva. EI riesgo de infección se multiplica por cinco (IC 95\%: 2,07-12,19) si la LM exclusiva dura menos de un mes, por 9,8 (IC 95\%: 4,06-23,66) si dura entre 30 y 60 días y por 3,4 (IC 95\%: 1,28-9,19) si dura entre 60 y 90 días.

Conclusiones: aunque muchos RN inician LM exclusiva, solo la tercera parte la mantiene a los cinco meses. Ningún factor se asoció a mayor duración de la misma. La $L M$ es un factor protector de IR cuando dura más de 90 días. Se debe fomentar el mantenimiento de la LM más allá del tercer mes, especialmente en aquellos con hermanos escolares.

Palabras clave: Lactantes. Infección respiratoria. Lactancia materna.

M. ${ }^{a}$ Mercedes Bueno Campaña, mbueno@fhalcorcon.es

Los autores declaran no presentar conflictos de intereses en relación con la preparación y publicación de este artículo. 
Breastfeeding and protection against respiratory tract infections in the first months of life

Abstract

Background: the protective role against respiratory infections is included among the advantages of breastfeeding (BF).

Objective: to describe the pattern of BF in a cohort of newborns and its protective role against respiratory infections (RI) in the infant.

Material and methods: a cohort of 316 newborns (NB) from Madrid Health Districts 8 and 9 was followed by telephone calls every fortnight during the winter season recording clinical and epidemiological data. Nasopharyngeal aspirate was obtained in every patient with symptoms compatible with RI.

Results: one thousand, eight hundred and sixty-five phone calls [median: 4.2 (111)], and 106 scheduled visits were conducted. At the age of 1 month $56.1 \%$ of the infants were exclusively breastfed, at 3 months $39.4 \%$ and at 5 months $31.9 \%$. The duration of BF was 65.1 days (SD: 43.49) and no factors were associated to a longer duration. Eighty nine acute $R I$ were registered. The presence of siblings in school age increased the risk of $R I$ by 1.74 (CI 95\%:1.12-2.72) in all the strata of duration of exclusive BF. The risk of $R I$ increased by 5 (Cl 95\%: 2.07-12.19) if breastfeeding lasted less than 1 month, by 9,8 (Cl 95\%: 4,06-23,66) between 30-60 days and by 3,4 (Cl 95\%: 1.28-9,19) if it lasted 60-90 days.

Conclusions: though many $N B$ begin $B F$, only a third part of them go on with it at the age of 5 months. No covariant was associated with a longer duration. BF is a protector factor against RI when it lasts more than 90 days. So it must be promoted specially in those infants with siblings in school age.

Key words: Infants. Respiratory tract infection. Breastfeeding.

\section{Introducción}

Las infecciones respiratorias (IR) son la primera causa de morbimortalidad en el lactante en nuestro medio. La Organización Nacional de la Salud (OMS) recomienda que todos los lactantes reciban lactancia materna (LM) exclusiva durante los seis primeros meses de vi$\mathrm{da}^{1}$. Se han comprobado sus beneficios, tanto en la disminución de la mortalidad como en la morbilidad en los países en vías de desarrollo ${ }^{2,3}$. También en países desarrollados, como Reino Unido ${ }^{4}$, España ${ }^{5}$, Australia ${ }^{6}$ EE. UU.7 o Países Ba- jos $^{8}$, se ha confirmado el papel de la LM en la disminución de las hospitalizaciones por infecciones gastrointestinales y respiratorias en lactantes sanos nacidos a término ${ }^{9}$. Sin embargo, el verdadero impacto de la LM en estos países no está bien delimitado. En el momento actual, el debate sobre la LM exclusiva se desarrolla entre su papel protector frente a infecciones y la insuficiente capacidad para satisfacer las necesidades nutricionales y energéticas del lactante más allá de los cuatro meses de vida ${ }^{10}$. Este debate está influido por el entorno 
socioeconómico en el que se lleven a cabo los estudios.

Las instituciones sanitarias tienen un papel trascendental en la promoción de la $L M$, facilitando a las mujeres las condiciones adecuadas para este fin, con ayudas económicas y prestaciones laborales al efecto y promocionando en las maternidades la LM con intervenciones como la Iniciativa Hospitales Amigos de los Niños.

El objetivo de nuestro trabajo es conocer el patrón de LM en nuestro medio y su eventual influencia en la incidencia de IR en lactantes en los primeros meses de vida.

\section{Material y métodos}

Estudio de cohortes prospectivo realizado entre los recién nacidos (RN) de los hospitales de las áreas sanitarias 8 y 9 de la Comunidad de Madrid (Hospital Universitario Fundación Alcorcón y Hospital Universitario Severo Ochoa de Leganés), reclutados del 1 de noviembre de 2005 al 31 de enero de 2006, por medio de una entrevista personal a los padres de todos los RN durante su estancia en la maternidad. El seguimiento se realizó desde el reclutamiento hasta el 30 de abril de 2006. Se solicitó consentimiento informado a los padres para su inclusión en el estudio. Para ello, debían estar dispuestos a acudir a las visitas de seguimiento cuando el niño presentara sintomatología respiratoria. Los criterios de exclusión se exponen en la tabla 1. El estudio fue aprobado por el Comité de Ética de los hospitales participantes.

Se trata de un proyecto de investigación encaminado a conocer el cumplimiento de la vacunación antigripal en el tercer trimestre de la gestación, la protección que proporcionaría a los recién nacidos en época gripal y las características clínicas y epidemiológicas de las IR en los primeros meses de vida ${ }^{11}$. Como objetivo secundario, se encontraba conocer el patrón de $L M$ en nuestro medio y la protección que pueda proporcionar en las IR. Los resultados de la primera parte del estudio, donde se des-

\section{Tabla 1. Criterios de exclusión de pacientes}

- Pretérmino de menos de 36 semanas de gestación

- Antecedente de patología grave neonatal: síndrome de dificultad respiratoria, sepsis, convulsiones, etc.

- Patología crónica de base: cardiopatía, nefropatía, epilepsia, etc.

- Dificultad para comunicación con los padres: por idioma, falta de teléfono disponible, etc.

- No facilidad de acceso al hospital 
criben las IR padecidas por la cohorte $y$ el alcance de la vacunación antigripal en la embarazada, han sido ya publica$\operatorname{dos}^{11,12}$. Remitimos al lector a dichos artículos para un conocimiento más detallado del diseño del estudio. Se presenta ahora la segunda parte del trabajo.

A los padres que aceptaron participar se les proporcionó un cuestionario con datos epidemiológicos (edad, nacionalidad, estudios, patología previa, número de hijos, estado vacunal, número de convivientes en el domicilio, hábito tabáquico) para que lo rellenaran antes de abandonar la maternidad. Se realizaron llamadas telefónicas quincenales hasta la finalización del seguimiento. En ellas, se interrogaba a los padres con un cuestionario prefijado sobre el patrón de lactancia y cambios en las características epidemiológicas (asistencia a guardería, número de convivientes, vacunación), y sobre la aparición de sintomatología respiratoria (fiebre, rinorrea, tos, anorexia, rechazo de la alimentación y dificultad respiratoria). Se concertaba una visita cuando referían síntomas compatibles con IR y se recogía una muestra de aspirado nasofaríngeo (ANF) si se cumplían al menos tres de los criterios preestablecidos expuestos en la tabla 2. Se proporcionó a los padres un teléfono de contacto para concertar la visita si los niños presentaban sintomatología entre las llamadas.

Se definió LM exclusiva cuando el lactante no había recibido ningún otro tipo de alimento distinto de la leche materna. Incluimos también como LM exclusiva lo que se conoce por lactancia materna predominante o casi exclusiva; es decir, los lactantes que han recibido algún biberón de forma esporádica y no de forma establecida.

Para el análisis del papel protector de la LM exclusiva, se consideró IR aquella que presentase tres o más de los criterios preestablecidos para la recogida de ANF, independientemente del resultado del estudio virológico.

\section{Tabla 2. Criterios de recogida de muestras}

- Fiebre: temperatura $>38^{\circ} \mathrm{C}$ rectal en menores de tres meses; $>38^{\circ} \mathrm{C}$ axilar en mayores de tres meses

- Rinorrea

- Tos

- Dificultad respiratoria

- Síntomas no respiratorios inespecíficos (deben cumplir al menos dos de los cinco): rechazo de las tomas (al menos tres tomas), vómitos (no regurgitaciones), diarrea, letargia o decaimiento, irritabilidad 
Ambos hospitales tienen Unidades de Pediatría dentro de un hospital general no terciario, se localizan en la zona sur de Madrid, y pertenecen a la Red Pública Sanitaria de la Comunidad Autónoma de Madrid. En el momento del estudio, ninguno estaba incluido en la Iniciativa Hospitales Amigos de los Niños.

El estudio virológico se llevó a cabo mediante determinación de reacción en cadena de polimerasa (PCR) en el ANF tomado a los niños cuando presentaron sintomatología respiratoria. Se realizó en el Laboratorio de Gripe y Virus Respiratorios del Instituto de Salud Carlos III, según la metodología previamente publicada ${ }^{12}$.

\section{Estudio estadístico}

Las variables cuantitativas se expresan como media (desviación estándar [DE]), (mediana y $\mathrm{P}_{25}-\mathrm{P}_{75}$, en el caso del número de llamadas, y duración del seguimiento) y las variables cualitativas como proporciones. Las características clínicas fueron comparadas mediante el test $\mathrm{t}$ de Student, el test U Mann-Whitney, el test chi-cuadrado y el test exacto de Fisher. Los valores de la $p$ son bilaterales. Se consideró estadísticamente significativo un valor de $p<0,05$. Se utilizó el modelo de riesgos proporcionales de Cox para analizar el efecto potencial de determinados factores epidemiológi- cos de las muestras sobre la presencia de IR. Estos se definieron mediante análisis univariable de los factores que habían demostrado previamente estar relacionados con las IR, incluyendo finalmente en el modelo los que demostraron asociación con un grado de significación $>0,2$. Las tasas de riesgo (HR) ajustadas se dan con intervalo de confianza del 95\% (IC 95\%). El análisis fue realizado empleando el Statistical Package of Social Science (SPSS) ${ }^{\oplus}$, versión 15.0.

\section{Resultados}

De un total de 568 RN elegibles ( 236 en Alcorcón y 332 en Leganés), 316 cumplían los criterios de inclusión y aceptaron participar en el estudio. Se completó el estudio en 294 (94\% de la muestra inicial) y se perdieron 22 casos. La causa más frecuente de pérdida $(11 / 22)$ fue no acudir a las citas programadas. Las características epidemiológicas de las madres y los datos perinatales de los RN se resumen en la tabla 3. No se encontraron diferencias entre ambas áreas en cuanto a dichas características. Se realizaron 1865 llamadas telefónicas (mediana: 4,2 por niño [P25: $\left.1 ; P_{75}: 11\right]$ ). La mediana de tiempo de seguimiento fue de 3,45 meses ( $\left.P_{25}: 2,95 ; P_{75}: 4,23\right)$. El $14,3 \%$ de los niños tenía más de cinco meses al terminar el estudio. 


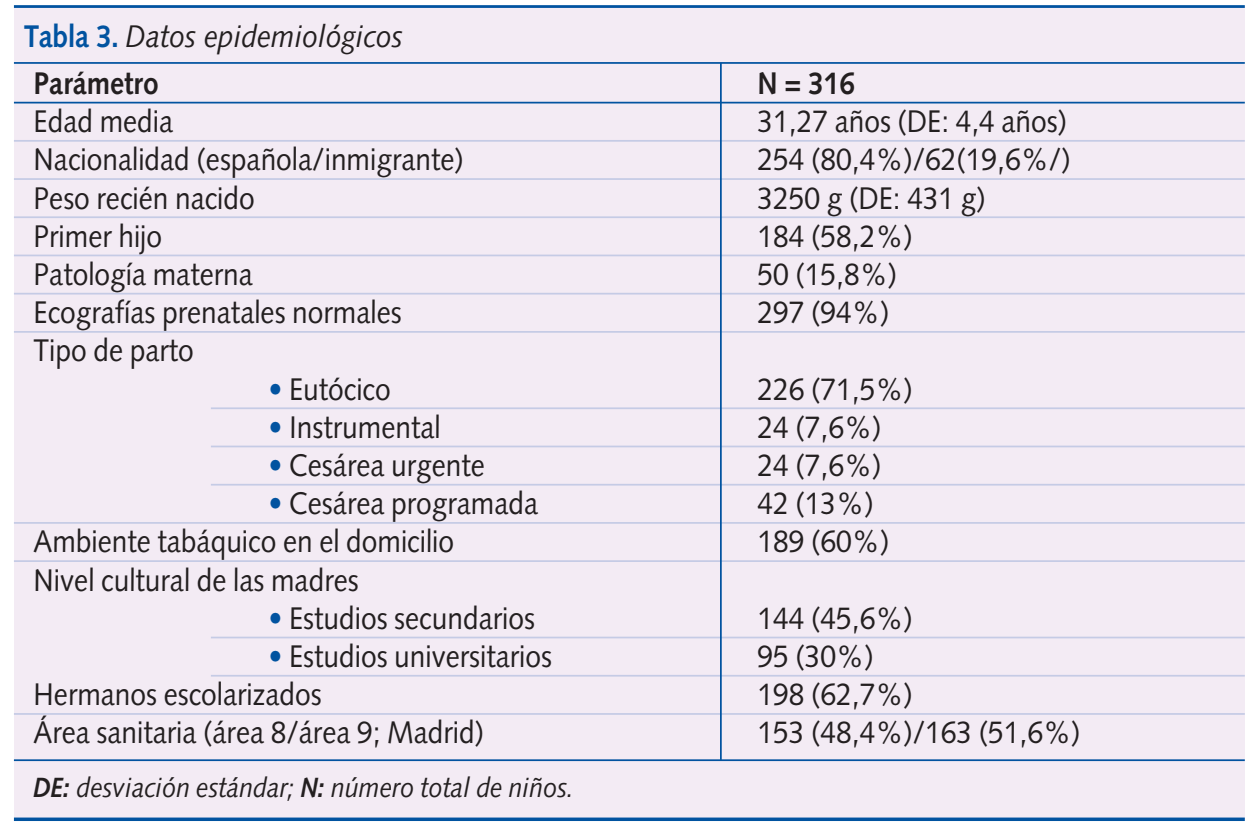

\section{Patrón de lactancia materna}

La evolución del patrón de LM se resume en la figura 1. A los 15 días de vida, el $66 \%$ de los niños recibía LM; el $18 \%$, mixta, y el $14 \%$, lactancia artificial (LA). A los dos meses, de los 286 niños seguidos hasta esa edad, el $45,8 \%$ mantenía LM exclusiva; el 32,2\%, mixta, y el $21 \%$, LA. A los cinco meses de edad, solo el $31,9 \%$ de los pacientes que se siguieron hasta esa edad (72) recibía LM de forma exclusiva, frente al $40 \%$ que recibía lactancia mixta y el $27,8 \%$ que recibía LA. No existían diferencias significativas en el patrón de lactancia en cuanto a edad de la madre, número de hijos, nacionalidad española o no, nivel de estudios o área sanitaria. La duración media de la LM en la muestra fue de 65,1 días (DE: 43,49).

\section{Infecciones respiratorias agudas}

Se concertaron un total de 106 visitas en 80 niños para la valoración clínica de la posible IR aguda. Se cumplieron criterios de IR aguda en 89 ocasiones (83,9\% de las visitas). El diagnóstico clínico más frecuente fue catarro de vías altas (82\%). Solo se detectó un virus en $17(23,2 \%)$ de las 73 muestras biológicas analizadas mediante PCR. El 15,5\% de los niños que realizaron visita por enfermedad precisó 
Figura 1. Evolución del patrón de lactancia.

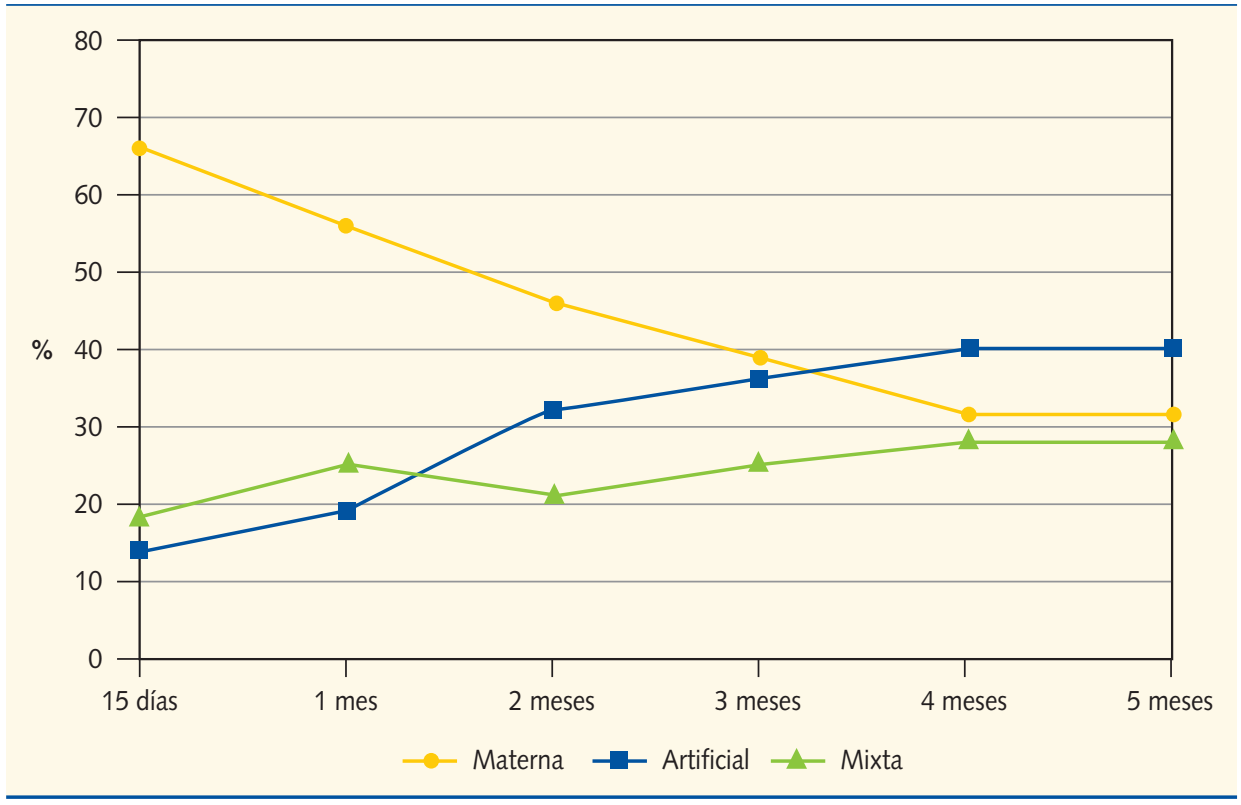

ingreso hospitalario. El virus aislado con mayor frecuencia fue el rinovirus (38\%), seguido del virus respiratorio sincitial $(33 \%)^{12}$.

\section{Lactancia materna e infecciones respiratorias agudas}

El $46 \%$ de las IR clínicas (con estudio virológico positivo o no) se produjo en niños menores de un mes. La edad media de los niños infectados fue de 45 días (DE: 26,14). La LM en los niños con IR se prolongó 61,75 días (DE: 40,30), frente a 66,10 días (DE: 44,38) que duró en los no infectados $(p=0,44)$. La duración media de la $L M$ en los niños que precisaron ingreso por su IR fue de 39 días (DE: 23,54), frente a 46,25 días (DE: 26,55$)$ en los niños infectados no ingresados. Estas diferencias no fueron significativas $(p>0,1)$. Se analizaron factores epidemiológicos considerados en otros estudios como asociados a IR (nacionalidad, estudios de la madre, hijos vivos, número de convivientes en el domicilio, fumadores y peso de recién nacido [PRN]) y no se relacionaron con un mayor riesgo de IR. Solo la presencia de hermanos en edad escolar mostró asociación con las IR. Al mes de vida, el $12,3 \%$ de los niños con hermanos en edad escolar se había infectado, frente al 
$8,2 \%$ de los que no los tenían. A los dos meses de edad, estas cifras ascienden al $28,5 \%$ y al $16,4 \%$, respectivamente. Estas diferencias resultaron significativas $(p=0,006)$. Tener hermanos en edad escolar multiplica por 1,74 la tasa de riesgo de IR en los primeros meses de vida (IC 95\%: 1,12-2,72). El tipo de lactancia en el momento de la infección no fue un factor de riesgo para IR (chi-cuadrado $=0,052 ; p=0,820$ ). Sin embargo, sí lo fue la duración de la misma. Si tomamos como referencia la duración de la LM exclusiva más de 90 días: el riesgo de infección se multiplica por cinco (IC 95\%: 2,073-12,19) si dura menos de un mes, por 9,8 (IC 95\%: 4,065$23,660)$ si dura entre 30-60 días y por 3,4 (IC 95\%: 1,280-9,198) si dura entre 60-90 días. Prolongar la LM exclusiva más de 90 días resultó un factor protector contra la IR y no existe ningún beneficio significativo cuando la lactancia duraba menos de 90 días. De esta manera, por cada cuatro niños (IC 95\%: 3,46$6,67)$ que reciben LM exclusiva más de 90 días se evitaría una IR. El análisis crudo de la tendencia de la asociación entre duración de la $L M$ y riesgo de IR demuestra una asociación negativa ( $z=-3,47 ; p=0,00051)$ que, una vez ajustada por la presencia de hermanos en edad escolar, continúa siendo signifi- cativa $(z=-3,36 ; p=0,0007)$. Es decir, la presencia de hermanos en edad escolar no modifica el efecto que la duración de la LM tiene sobre el riesgo de IR. Los niños con hermanos en edad escolar se infectan más en todos los estratos de duración de la LM exclusiva, pero en los que la LM dura más de 90 días, las infecciones disminuyen en ambos grupos.

\section{Discusión}

El patrón de lactancia que describimos en nuestro estudio es similar al comunicado en otras publicaciones basadas en resultados obtenidos en una población con características socioeconómicas y culturales similares ${ }^{5,13}$. Nuestros resultados también muestran que la LM exclusiva no protege de las IR leves en los primeros meses de vida, aunque sí se ha mostrado como un factor protector cuando se prolonga más de 90 días. Aunque se observa una tendencia a proteger de las infecciones más graves, representadas por aquellas que precisaron ingreso hospitalario, ni el tamaño de la muestra ni quizás la duración del seguimiento permitieron establecer resultados significativos. La presencia de hermanos en edad escolar resulta un factor de riesgo de presentar IR clínica en los primeros meses de edad, aunque su efecto se ve amortiguado de forma 
significativa en los niños con LM exclusiva durante más de 90 días, respecto a otros patrones de menor duración.

En una revisión de la Cochrane del año 2002, recientemente actualizada ${ }^{14}$, respecto al beneficio potencial que tendría la LM exclusiva mantenida seis meses con respecto a cuatro meses, se apuntó una disminución en las enfermedades de origen intestinal en aquellos que recibieron LM un tiempo más prolongado, incluso en los países desarrollados. Sin embargo, no existe ningún trabajo serio que demuestre que ocurra algo similar con las IR, hay resultados contradictorios, sobre todo en los países de nuestro entorno.

En el año 2001, la OMS cambió su recomendación sobre la LM exclusiva de cuatro a seis meses, en parte sobre la base del estudio desarrollado en Bielorrusia (considerado en el estudio como país desarrollado ${ }^{15}$ que apuntó un importante descenso de las infecciones gastrointestinales en relación con la duración de la $\mathrm{LM}$ exclusiva. Pero tampoco este estudio consiguió demostrar una disminución de las $I R$, y hay que resaltar que, pese a tratarse de un trabajo muy bien diseñado, con una muestra amplia y con un sistema sanitario comparable con el de otros países occidentales, algunas características de la población es- tudiada eran diferentes a las de la población de los países más desarrollados, lo que hace difícil extender a nuestro medio los resultados obtenidos. No existen guarderías, y la baja maternal dura tres años, lo que elimina un factor reconocido de IR en estos niños.

En 2005, se publicó un trabajo realizado en Atención Primaria ${ }^{13}$ en España, en el que se analizaba de forma retrospectiva la presencia de IR o digestivas, independientemente de si necesitaron hospitalización; se encontró que, de forma global, la LM no protegía frente a estas infecciones, aunque si se separaban por trimestres parecía observarse un factor protector en el segundo trimestre de vida. No se hace referencia a qué estación del año corresponde ese segundo trimestre, que podría ser un factor de sesgo importante. Nuestros pacientes fueron seguidos durante la estación invernal.

Más adelante, en el año 2006 Chantry et al. ${ }^{7}$ desarrollaron en EE. UU. un estudio que demuestra que la LM exclusiva mantenida al menos seis meses también protege de la neumonía y de la otitis media recurrente, pero no de otras IR.

Si nos centramos en países en vías de desarrollo, del análisis de los trabajos realizados $^{16,17}$ se desprende que la $L M$ exclusiva protege claramente de la mor- 
talidad por IR, frente a la LA. Se ha visto que incluso la lactancia mixta disminuye ese riesgo. Probablemente, estos resultados aparentemente contradictorios con respecto a lo mencionado anteriormente se deban a que los planteamientos utilizados son diferentes. En estos países, el objetivo es evaluar la mortalidad producida por procesos respiratorios y gastrointestinales ${ }^{18,19}$. En los países desarrollados $s^{5-7,9,20}$, el objetivo a considerar es la hospitalización por IR.

Nuestros resultados no nos permiten obtener conclusiones en cuanto a la duración óptima de la LM exclusiva a efectos de protección de IR, aunque sí corroboran en cierto modo lo propuesto por la OMS, ya que no se demuestra una protección contra IR en los primeros meses de vida. Solo cuando la LM se mantiene más de 90 días se observa un claro beneficio sobre la incidencia de IR.

Nuestro trabajo es uno de los primeros estudios prospectivos que analizan la incidencia de IR de cualquier tipo en relación con la LM exclusiva. También se estudiaron otros factores de riesgo conocidos para la aparición de IR (ambiente tabáquico, origen étnico, hermanos en domicilio, nivel cultural, etc.) y no se han encontrado diferencias significativas con respecto a ninguno, salvo la presencia en el domicilio de hermanos escolarizados.
Entre las fortalezas de nuestro estudio se encuentra sin duda su diseño, con llamadas quincenales para encuesta epidemiológica, de forma que eliminamos el sesgo de memoria ${ }^{4,5}$, o de mayor grave$\mathrm{dad}^{6}$, y detectamos incluso las infecciones más leves. El seguimiento de los niños fue exhaustivo. Esto, junto con los criterios definidos para la recogida de muestras, hace que nuestro estudio sea una muestra fiable del alcance real de las IR en este grupo de edad y de las características epidemiológicas que las rodean. Aunque nuestro objetivo no eran las infecciones gastrointestinales, cabe resaltar que dentro del cuestionario que se realizaba se incluían manifestaciones digestivas como dato asociado a IR, y en ningún caso se encontraron estos síntomas.

Entre las debilidades, quizás la más importante sea la duración del seguimiento y el relativo pequeño número de niños seguidos. Posiblemente, de haberse prolongado en el tiempo podría haber puesto de manifiesto la verdadera magnitud de la protección que la LM puede proporcionar, como ha ocurrido en otros trabajos ${ }^{4,7}$. Otro aspecto negativo de nuestro estudio es el escaso número de muestras respiratorias positivas que obtuvimos con respecto a las muestras recogidas. Se puede pensar que los criterios para diagnosticar IR fueron 
muy laxos, pero el objetivo era detectar todas las infecciones, aunque su manifestación clínica fuera leve.

En resumen, en nuestro medio, un porcentaje elevado de RN inicia la alimentación mediante LM exclusiva; sin embargo, alrededor de los dos meses de vida un alto porcentaje la abandona. Ninguno de los factores epidemiológicos considerados se relacionó con la prolongación de LM. Solo la existencia de hermanos en edad escolar en el domicilio resultó ser un factor de riesgo asociado a la presencia de IR en los lactantes de corta edad, independientemente del tipo de lactancia recibido. Aunque el tipo de lactancia en el momento de la infección no influye sobre el riesgo de IR, sí lo hace su duración, de manera que cuando se prolonga más de tres meses disminuye de forma significativa el riesgo de IR en los meses posteriores, respecto a periodos más cortos de LM exclusiva. Este efecto beneficioso se mantiene incluso en presencia de hermanos en edad escolar, por lo que debería fomentarse la prolongación de la LM exclusiva más allá del tercer mes de vida, especialmente en aquellos niños con hermanos escolarizados.

\section{Agradecimientos}

Los autores quieren manifestar su agradecimiento a Dolores Martín Ríos, del Departamento de Medicina Preventiva del Hospital Universitario Fundación Alcorcón, por su inestimable ayuda en el análisis estadístico de este proyecto.

\section{Bibliografía}

1. World Health Organization. Global Strategy for Infant and Young Child feeding. Geneva, Swizerland: World Health Organization; 2002.

2. WHO. Collaborative Study Team on the Role of Breastfeeding on the prevention of infant mortality due to infectious deseases in less developed countries: a pooled analysis. Lancet. 2000;355: 451-5.

3. Bahl R, Frost C, Kirkwood BR, Martinez J, Bhandari N, Arthur P. Infant feeding patterns and risks of death and hospitalization in the first half of infancy: multicentre cohort study. Bull WHO. 2005;83:418-26.

4. Quigley MA, Kelly YJ, Sacker A. Breastfeeding and hospitalization for diarrheal and respiratory infection in the United Kingdom: Millenium Cohort Study. Pediatrics. 2007;119:e837-42.

5. Paricio Talayero JM, Lizán-García $M$, Otero Puime A, Benlloch Muncharanz MJ, Beseler Soto B, Sánchez-Palomares $M$ et al. Full breastfeeding and hospitalization as a results of infections in the first year of life. Pediatrics. 2006;118:e92-9.

6. Oddy WH, Sly PD, de Klerk NH, Landau LI, Kendall GE, Holt PG et al. Breast feeding and respi- 
ratory morbidity in infancy: a birth cohort study. Arch Dis Child. 2003;88:224-8.

7. Chantry CJ, Howard CR, Auinger P. Full Breastfeeding Duration and Associated Decrease in Respiratory Tract Infection in US Children. Pediatrics. 2006;117;425-32.

8. Duijts L, Jaddoe VW, Hofman A, Moll HA. Prolonged and Exclusive Breastfeeding Reduces the Risk of Infectious Diseases in Infancy. Pediatrics. 2010;126;e18-25.

9. Bachrach VR, Schwarz E, Bachrach LR. Breastfeeding and the Risk of Hospitalization for Respiratory Disease in Infancy. A Metaanalysis. Arch Pediatr Adolesc Med. 2003;157:237-43.

10. Kramer M, Matush L, Bogdanovich $N_{1}$ Aboud F, Mazer B, Fombonne E et al. Health and development outcomes in 6.5-y-old children breastfed exclusively for 3 or 6 mo. Am J Clin Nutr. 2009;90:1070-4.

11. Bueno Campaña $M$, González Spinola $A$, Parra Cuadrado E, Quevedo Teruel S, Calvo Rey C. Vacunación antigripal en la embarazada. Prog Obstet Ginecol. 2010;53:293-6.

12. Bueno Campaña $M$, Calvo Rey $C$, Vázquez Álvarez MC, Parra Cuadrado E, Molina Amores C, Rodrigo García G, y cols. Infecciones de vías respiratorias en los primeros 6 meses de vida. An Pediatr (Barc). 2008;69:400-5.

13. Carratalá Munuera MC, Gascón Pérez E, Raga Ortega M. ¿Es la lactancia materna un factor de protección ante los procesos infecciosos? Estudio de casos y controles. Aten Primaria. 2005; 35(3):140-5.
14. Kramer MS, Kakuma R. Optimal duration of exclusive breastfeeding. [Systematic Review] Cochrane Pregnancy and Childbirth Group Cochrane Database of Systematic Reviews. 3, 2008.

15. Kramer MS, Chalmers B, Hodnett ED, Sevkovskaya Z, Dzikovich I, Shapiro S et al. A Randomized Trial in the Republic of Belarus Promotion of Breastfeeding Intervention Trial (PROBIT). JAMA. 2001;285:413-20.

16. Zaman K, Baqui AH, Yunus MD, Bateman OM, Chowdhury HR, Black RE. Acute respiratory infections in children: a community- based longitudinal study in rural Bangladesh. J Trop Pediatr. 1997;43:133-7.

17. Bertran AP, de Onis M, Lauer JA, Villar J. Ecological study of effect of breastfeeding on infant mortality in Latin America. BMJ. 2001;323: 303-6.

18. Arifeen S, Black RE, Antelman $G$, Baqui $A$, Caulfield L, Becker S. Exclusive Breastfeeding Reduces Acute Respiratory Infection and Diarrhea Deaths Among Infants in Dhaka Slums. Pediatrics. 2001;108:e67.

19. Kristensen IA, Olsen J. Determinants of acute respiratory infections in Soweto: a population based birth cohort. S Afr Med J. 2006;96: 633-40.

20. Pardo Crespo R, Pérez Iglesias F, Llorca J, Álvarez Granda L, García Fuentes M, Martínez González MÁ et al. Breast-feeding and risk of hospitalization for all causes and fever of unknown origin. Eur J Pub Health. 2004;14:230-4. 